\title{
Certification of Smart-Card Applications in Common Criteria
}

Proving Representation Correspondences

\author{
Iman Narasamdya ${ }^{1}$ and Michaël Périn ${ }^{2, \star}$ \\ ${ }^{1}$ FBK-Irst, Italy \\ narasamdya@fbk. eu \\ ${ }^{2}$ Verimag - UJF, France \\ Michael.Perindimag. fr
}

\begin{abstract}
We present a method for proving representation correspondences in the Common Criteria (CC) certification of smart-card applications. For security policy enforcement, the $\mathrm{CC}$ defines a chain of requirements: a security policy model (SPM), a functional specification (FSP), and a target-of-evaluation design (TDS). In our approach to the CC certification, these requirements are models of applications that can have different representations. A representation correspondence (RCR) describes a correlation between the representations of two adjacent requirements. One task in the $\mathrm{CC}$ certification is to demonstrate formal proofs of RCRs. We first develop a modelling framework by which the representations of SPM, FSP and TDS can be described uniformly as models of an application. We then define RCRs as mutual simulations between two application models over sets of observable events and variables. We describe a proof technique for proving RCRs and providing certificates about them based on assertions relating two models at specific locations. We show how RCRs can help us prove property preservation from the SPM to the FSP and the TDS.
\end{abstract}

\section{Introduction}

We describe in this paper our work on developing a method for formal certification of smart-card applications in the framework of Common Criteria (CC) [1]. This work is part of an industrial project called EDEN2 1 The CC is an international standard for the evaluation of security related systems. It guarantees that a target of evaluation (TOE), or a system, enforces security policies by means of an assurance architecture. For assurances in the development process, this architecture consists of a chain of requirements starting from the model of the policies at the start of the chain, to the low-level design and the implementation of the system at the end of the chain.

At the highest level of the CC certification, which is called evaluation assurance level 7 (or EAL7), the following chain of requirements are needed in the assurance architecture: (1) a formal security model (SPM), (2) a formal functional specification

\footnotetext{
${ }^{\star}$ This research has been supported by funding from RNTL EDEN project.

${ }^{1}$ Research and industrial partners include Verimag, CEA, Gemalto, and Trusted-Logic; see http : / / www . eden-rntl . org
} 
of security functions (FSP), and (3) a TOE design (TDS). The SPM models the policy independently of the implementation, the FSP describes input-output relationships of security functions, and the TDS is a low-level design that is close to the implementation. A representation correspondence (RCR) demonstrates the correlation between each two adjacent requirements in the chain. The CC EAL7 certification consists of proving that the SPM, the TDS, and the FSP satisfy the security policies, and providing certificates about this satisfaction. In addition, the CC EAL7 also requires formal proofs of RCRs between the SPM and the FSP, and between the FSP and the TDS.

In this paper we are concerned with proving RCRs and providing certificates about them. We present a method for proving RCRs in the context of smart-card applications. First, we develop a framework for modelling smart-card applications such that the formal models capture the operations of the applications, in particular our model allows one to reason about card tears (or power loss) and transaction mechanism that are present in smart-card applications. In this framework, a model of an application consists of a set of command procedures (or simply command). Each command is presented by two transition graphs (or control-flow graphs), one describes the normal behavior of the command and the other describes what the command has to perform when a card tear occurs. The FSP and the TDS are essentially models of an application. In EDEN2, the SPM consists of two entities: one entity is a model of the application and the other is a set of assertions (or formulas) in some logic such that the assertions describe security properties. In the sequel, we refer to the former entity when we speak about SPM. Card readers communicate with a smart-card application by sending a sequence of commands. We model this interaction with a main procedure that takes as the only input a sequence of commands, and for each command, the procedure calls the corresponding command procedure in the application. The semantics of an application is then characterized by the set of the main procedure's runs.

We define RCRs between two application models as bisimulation equivalence consisting of mutual simulations between the models over observable events and variables. To this end, given two models $S$ and $T$ of an application, we associate with $S$ and $T$ the same set of observable events and for each event we associate a mapping between observable variables. Intuitively, we say that there is an RCR between $S$ and $T$ if for every run of $S$, there is a run of $T$ on the same input, and vice versa, such that (1) both runs exhibit the same sequence of observable events, and (2) for each two equal events, the values of corresponding observable variables coincide. Having a unified model for smart-card applications allows us to have only a single definition of RCRs such that the definition is applicable for RCRs between the SPM and the FSP, and between the FSP and the TDS. Furthermore, we will show that our definition of RCR helps us prove property preservation from one model to the other. That is, as required by the CC EAL7 certification, the RCRs must guarantee that all security properties satisfied by the SPM are satisfied by the FSP and the TDS.

We develop a proof technique for proving RCRs. We prove RCRs between $S$ and $T$ by proving the RCR between each corresponding commands in $S$ and $T$. We apply a theory of inter-program properties described in [16] to proving RCRs. Interprogram properties are properties relating two programs. RCRs are essentially interprogram properties. We prove RCRs by using assertions that describe data abstraction 
and control mapping between the transition graphs of the corresponding commands. The theory also provides a notion of certificate about inter-program properties. Such a certificate is essential to the CC EAL7 certification.

Proving RCRs are challenging due to nontrivial data abstractions between application models and due to language features in which the models are written. Consider a command checkPIN used to authenticate users by checking an input PIN against the PIN stored on the card. The security policy does not require the PIN to be in some specific format. Thus, in the SPM the PIN can simply be a natural number. For security, the command uses variables trial as a trial-remaining counter. If the input PIN does not match the stored PIN, then trial is decremented, and if it gets 0 , then the PIN is blocked. In the FSP, developers usually take defensive measures. The PIN in the FSP is now an array of natural numbers, and prior to checking the input PIN, the variable trial must be decremented. We then have the following excerpts of over-simplified checkPIN, the SPM and the FSP are on the lefthand and righthand, respectively:

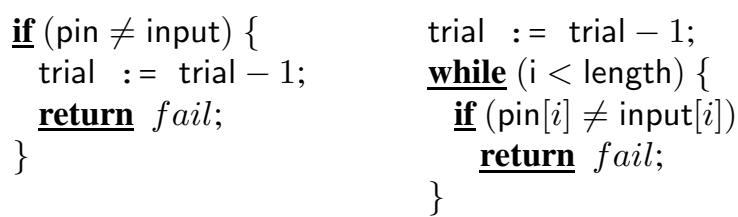

If we associate an event with every update of trial, then in the SPM this event occurs at the end of command execution, but in the FSP it occurs at the beginning. Thus, we may end up with different sequences of observable events. This poses some difficulties in determining observable events in RCRs. Note that in the SPM and the FSP above, the data abstraction introduces a loop in the FSP. To prove that for every run of the SPM there is a "corresponding" run of the FSP, one has to prove that the loop will not yield non-terminating run. We will show later that in the presence of transaction mechanism, we sometimes have to relax the definition of RCR. That is, we only require that for every run of the TDS, there is a corresponding run of the FSP.

In summary the contributions of this paper is a method for proving representation correspondences as a part of the CC EAL7 certification of smart-card applications.

The outline of this paper is the following. We first discuss our framework for formally modelling smart-card applications. We then develop a notion of representation correspondence based on this framework. Afterward we describe briefly the theory of inter-program properties. Then, we discuss our proof technique for proving RCRs based on the theory. We then show how RCRs allow us to preserve property in the chain of the $\mathrm{CC}$ requirements. Finally, we discuss some related work and conclude this paper.

\section{Formal Models and Representation Correspondences}

\subsection{Transition Graphs and Computation Sequences}

A smart-card application is a program consisting of $m+1$ procedures: $\operatorname{main}, c_{1}, \ldots, c_{m}$, where main is the main procedure and $c_{1}, \ldots, c_{m}$ are command procedures. In the sequel, command procedures are often called commands. Each procedure $P$ consists of a finite set of program points and is presented as two disjoint transition graphs (or 
program-point flow graphs) $\mathbf{G}_{P}^{n}$ and $\mathbf{G}_{P}^{a}$. A transition graph is a finite directed graph whose nodes are program points. Each edge of a transition graph is labelled with a guard, an assignment instruction, a goto instruction (or a skip instruction), or a procedure call. The transition graph $\mathbf{G}_{P}^{n}$ describes the normal behavior of $P$, while the transition graph $\mathbf{G}_{P}^{a}$ describes what the application has to do when a card tear occurs during the execution of $P$.

We assume that every transition graph $\mathbf{G}_{P}$ has a unique entry point, denoted by $\operatorname{entry}\left(\mathbf{G}_{P}\right)$ and a unique exit point, denoted by $\operatorname{exit}\left(\mathbf{G}_{P}\right)$. As such, every procedure $P$ has a unique entry point $\operatorname{entry}(P)=\operatorname{entry}\left(\mathbf{G}_{P}^{n}\right)$, and two exit points, normal exit point exit $_{n}(P)=\operatorname{exit}\left(\mathbf{G}_{P}^{n}\right)$ and abrupt exit point exit $(P)=\operatorname{exit}\left(\mathbf{G}_{P}^{a}\right)$.

The main procedure takes as input a sequence of input commands. In turn, the procedure reads each input command of the form $(C, \bar{v})$, where $C$ is the command name and $\bar{v}$ are the input values for $C$. For each input command $(C, \bar{v})$, the main procedure calls the corresponding command $C$ on input $\bar{v}$, or call $C(\bar{v})$.

We introduce a restriction on command procedures, that is, for every command procedure $P$, the graphs $\mathbf{G}_{P}^{n}$ and $\mathbf{G}_{P}^{a}$ do not contain edges labelled with procedure calls. Similarly, the graph $\mathbf{G}_{\text {main }}^{a}$ does not contain such edges. This restriction does not limit the applications that can be modelled in our framework. Procedures called by command procedures in smart-card applications are usually not recursive and thus can be inlined. For technical reason, we assume that, for every command procedure, $\mathbf{G}_{\text {main }}^{n}$ contains an edge labelled with a call to the procedure.

We describe the run-time behavior of an application as sequences of configurations. A configuration of a run is a pair $(p, \sigma)$ where $p$ is a program point and $\sigma$ is a state mapping variables to values. Given a procedure $P$, a configuration $(p, \sigma)$ is called an entry configuration for $P$ if $p$ is an entry point of $P$, a normal exit configuration for $P$ if $p$ is a normal exit point of $P$, and an abrupt exit configuration for $P$ if $p$ is an abrupt exit point of $P$

The semantics of an application is defined as a transition relation with transitions of the form $\left(p_{1}, \sigma_{1}\right) \stackrel{l}{\longmapsto}\left(p_{2}, \sigma_{2}\right)$, where $\left(p_{1}, \sigma_{1}\right)$ and $\left(p_{2}, \sigma_{2}\right)$ are configurations and $l$ is a transition label. Transitions are of the following kinds:

- Intra-graph transition, where the pair $\left(p_{1}, p_{2}\right)$ is an edge of a transition graph, $l$ is the label of the edge such that $l$ is not a procedure call.

- Call and return transitions, where $l$ is a procedure call and a special label ret, respectively.

- Abrupt transition, where $p_{1}$ is in $\mathbf{G}_{P}^{n}, p_{2}$ is entry $\left(\mathbf{G}_{P}^{a}\right), l$ is a special label $a b$, and $\sigma_{1}=\sigma_{2}$.

We allow labels of transitions (or edges of transition graphs) to be associated with events, which means that the transitions emit the events. We will use a special event variable $\varepsilon$ to store emitted events. That is, if a transition emits an event $E$, then it is the same as an assignment of $E$ to $\varepsilon$. Details of transition relations are in [12].

We use the following assumptions for transition relations. First, for every procedure $P$, every point $p$ in $\mathbf{G}_{P}^{n}$, and every state $\sigma$, there is a transition $(p, \sigma) \stackrel{l}{\longmapsto}\left(\operatorname{entry}\left(\mathbf{G}_{P}^{a}\right), \sigma\right)$. That is, a card tear can occur non-deterministically. Second, there is no transition from an exit configuration $(p, \sigma)$, where $p=\operatorname{exit}_{a}(P)$ for every procedure $P$, or $p=\operatorname{exit}_{n}($ main $)$. Third, intra-graph transitions are deterministic. Forth, transitions are atomic. 
A computation sequence of an application $A$ is either a finite or an infinite sequence of

$$
\left(p_{0}, \sigma_{0}\right) \stackrel{l_{1}}{\longmapsto}\left(p_{1}, \sigma_{1}\right) \stackrel{l_{2}}{\longmapsto}\left(p_{2}, \sigma_{2}\right) \ldots
$$

where, for all $i$, the transition $\left(p_{i}, \sigma_{i}\right) \stackrel{l_{i+1}}{\longmapsto}\left(p_{i+1}, \sigma_{i+1}\right)$ is justified by a transition in the transition relation of $A$. When a computation sequence is finite, then it ends with a configuration. A run of a procedure $P$ in $A$ from a state $\sigma_{0}$ is a computation sequence of $A$ such that $p_{0}=\operatorname{entry}(P)$. For every run of a command procedure $P$, the run terminates when it reaches an exit configuration for $P$, and can only terminate in such a configuration. We say that the run terminates normally (terminates abruptly) if the final configuration is a normal (abrupt) exit configuration for $P$. A run of an application $A$ from a state $\sigma$ is a run of the procedure main from $\sigma$. Especially for main, a run of main terminates normally if the final configuration is a normal exit configuration for main, and terminates abruptly if the final configuration is an abrupt exit configuration for any procedure. A run of a transition graph $G$ in an application $A$ is a computation sequence of $A$ such that $p_{0}=\operatorname{entry}(G)$ and for all $i$, the pairs $\left(p_{i}, p_{i+1}\right)$ is an edge of the graph.

\subsection{Representation Correspondences}

For our discussion on representation correspondences (RCRs), we assume that we are given two models $S$ and $T$ of an application, where $T$ is an implementation of $S$. That is, $S$ and $T$ can be, respectively, an SPM and an FSP, or they can be, respectively an FSP and a TDS. For simplicity, we assume that each command in $S$ has a corresponding command, with the same name, in $T$, and vice versa. We assume further that $S$ and $T$ have disjoint sets of transition graphs and disjoint sets of variables.

To define RCRs, we associate with both $S$ and $T$ the same set of observable events, and for each observable event we associate a one-to-one correspondence between observable variables of $S$ and $T$ at the start or final configurations of the transitions that emit the event. Intuitively, there is an RCR between $S$ and $T$ if for every run of $T$, there is a run of $S$ on the same input, such that (1) both runs terminate or generate infinite computation sequences, (2) these runs exhibit the same sequence of observable events, (3) the values of corresponding observable variables in the configurations of each corresponding events coincide, and (4) vice versa for every run of $S$.

We first discuss the set of observable events. For every procedure $P$, we associate every incoming edge into $\operatorname{exit}_{n}(P)$ with either a Pass $_{P}$ or a Fail $P$ events. The first event denotes a successful completion of a run of $P$, while the latter denotes a logic failure. We associate every incoming edge into $\operatorname{exit}_{a}(P)$ with an $\operatorname{Abrupt}_{P}$ event and every call transition to a procedure $P$ with a Call $P$ event.

Next, we associate one-to-one correspondences between observable variables for events. For each command procedure $P$ and for every configuration $\gamma$ such that there is a configuration $\gamma^{\prime}$ and $\gamma^{\prime} \stackrel{l}{\longmapsto} \gamma$ where $l$ is associated with $\operatorname{Pass}_{P}$, we associate with $\gamma$ a set $O_{S}$ of observable variables if $\gamma$ belongs to an $S$ 's run, and a set $O_{T}$ if $\gamma$ belongs to a $T$ 's run, such that there is a one-to-one correspondence $O b s$ between $O_{S}$ and $O_{T}$. Similarly for $l$ associated with Fail $_{P}$ and Abrupt $_{P}$. When $l$ is associated with 
Call $P$, then, instead of $\gamma$, we associate $O_{S}$ and $O_{T}$ with $\gamma^{\prime}$ such that if the parameters of $P$ in $S$ and in $T$ are, respectively, $\bar{x}=x_{1}, \ldots, x_{m}$ and $\bar{y}=y_{1}, \ldots, y_{n}$, then $m=n,\left\{x_{1}, \ldots, x_{m}\right\} \subseteq O_{S}$ and $\left\{y_{1}, \ldots, y_{n}\right\} \subseteq O_{T}$, and Obs maps $x_{i}$ to $y_{i}$ for all $i=1, \ldots, m$. We also associate entry configurations of main with the sets $O_{S}$ and $O_{T}$ such that the input variables of $S$ and $T$ are mapped to each other.

We associate observation function $\mathcal{O}$ with each $S$ and $T$ to identify observable configurations and transition labels. That is, for a configuration $\gamma$, the function $\mathcal{O}(\gamma)=\gamma$ if $\gamma$ is associated with a set of observable variables, otherwise $\mathcal{O}(\gamma)=\perp$. Similarly, for a label $l$ of a transition, $\mathcal{O}(l)=e$ if $l$ emits an observable event $e$, otherwise $\mathcal{O}(l)=\perp$. An observation sequence of a computation sequence $R$, denoted by $o(R)$, is obtained by turning $R$ into an alternating sequence of configurations and transition labels, and applying the observation function $\mathcal{O}$ to each configuration and transition label of $R$. That is, for a computation sequence $R=\gamma_{0} \stackrel{l_{1}}{\longmapsto} \gamma_{1} \stackrel{l_{2}}{\longmapsto} \gamma_{2} \stackrel{l_{3}}{\longmapsto} \ldots$, we have $o(R)=\mathcal{O}\left(\gamma_{0}\right), \mathcal{O}\left(l_{1}\right), \mathcal{O}\left(\gamma_{1}\right), \mathcal{O}\left(l_{2}\right), \mathcal{O}\left(\gamma_{2}\right), \mathcal{O}\left(l_{3}\right), \ldots$ A $\perp$-free observation sequence of a computation sequence $R$, denoted by $o_{\perp}(R)$ is obtained from $o(R)$ by suppressing $\perp$ in $o(R)$.

We say that two states $\sigma_{1}$ and $\sigma_{2}$ are compatible with respect to a one-to-one correspondence $O b s$ between the sets $O_{1}$ and $O_{2}$ of observable variables in the domain of, respectively, $\sigma_{1}$ and $\sigma_{2}$ if for every $x \in O_{1}$, we have $\sigma_{1}(x)=\sigma_{2}(\operatorname{Obs}(x))$. Two configurations $\gamma_{1}=\left(p_{1}, \sigma_{1}\right)$ and $\gamma_{2}=\left(p_{2}, \sigma_{2}\right)$ are compatible if there are sets $O_{1}$ and $\mathrm{O}_{2}$ of observable variables associated with $\gamma_{1}$ and $\gamma_{2}$ such that (1) there is a one-to-one correspondence $O b s$ between $O_{1}$ and $O_{2}$, and (2) $\sigma_{1}$ and $\sigma_{2}$ are compatible with respect to $O b s$.

DEFINITION 2.1. We say that two computation sequences $R_{1}$ and $R_{2}$ are observationally equivalent (or stuttering equivalent) if, let

$$
o_{\perp}\left(R_{1}\right)=\theta_{1}, \theta_{2}, \ldots \quad o_{\perp}\left(R_{2}\right)=\theta_{1}, \theta_{2}^{\prime}, \ldots,
$$

$o_{\perp}\left(R_{1}\right)$ and $o_{\perp}\left(R_{2}\right)$ are of the same length, and for all $i$, we have either (1) $\theta_{i}=\gamma$ and $\theta_{i}^{\prime}=\gamma^{\prime}$, for configurations $\gamma$ and $\gamma^{\prime}$, such that $\gamma$ and $\gamma^{\prime}$ are compatible, or (2) $\theta_{i}=\theta_{i}^{\prime}$.

DEFINITION 2.2. There is a representation correspondence between a procedure $P$ of $S$ and a procedure $P^{\prime}$ of $T$ if for every run $R$ of $P$ from a configuration $\gamma$, there is a run $R^{\prime}$ of $P^{\prime}$ from a configuration $\gamma^{\prime}$, where $\gamma$ and $\gamma^{\prime}$ are compatible, and vice versa, such that $R$ and $R^{\prime}$ are observationally equivalent.

There is a representation correspondence between $S$ and $T$ if there is a representation correspondence between main of $S$ and main of $T$.

In the above definition, due to call transitions and our assumption that $\mathbf{G}_{\text {main }}^{n}$ contains at least a call edge for every command procedure, the configurations $\gamma$ and $\gamma^{\prime}$ have sets of observable variables associated with them. Note that to have $\gamma$ and $\gamma^{\prime}$ compatible, then the procedures $P$ and $P^{\prime}$ must refer to the same command. The notion of RCR for procedures is useful for proving RCR between $S$ and $T$. Since main can be thought of as a loop that read input command and call the command, then proving RCR between $S$ and $T$ can be reduced to proving RCR between each corresponding commands. 


\section{Theory of Inter-Program Properties}

In this section we describe an abstract theory for describing and proving properties that relate two programs, or inter-program properties. A detailed description of the theory can be found in [16]. The theory deals with programs that are represented as transition graphs described in the previous section.

For describing and proving inter-program properties, the theory considers two programs $P_{1}$ and $P_{2}$ as a pair $\left(P_{1}, P_{2}\right)$, such that they have disjoint flow graphs and disjoint sets of variables. A state $\sigma$ for the pair $\left(P_{1}, P_{2}\right)$ can be considered as a pair $\left(\sigma_{1}, \sigma_{2}\right)=\sigma$, such that $\sigma_{1}$ is for $P_{1}$ and $\sigma_{2}$ is for $P_{2}$. A configuration is a tuple $\left(p_{1}, p_{2}, \sigma_{1}, \sigma_{2}\right)$ such that $\left(p_{1}, \sigma_{1}\right)$ is a configuration for $P_{1}$ and $\left(p_{2}, \sigma_{2}\right)$ is a configuration for $P_{2}$. The semantics of $\left(P_{1}, P_{2}\right)$ is a transition relations containing two kinds of transitions:

1. $\left(p_{1}, p_{2}, \sigma_{1}, \sigma_{2}\right) \mapsto\left(p_{1}^{\prime}, p_{2}, \sigma_{1}^{\prime}, \sigma_{2}\right)$, such that $\left(p_{1}, \sigma_{1}\right) \mapsto\left(p_{1}^{\prime}, \sigma_{1}^{\prime}\right)$ is in $P_{1}$;

2. $\left(p_{1}, p_{2}, \sigma_{1}, \sigma_{2}\right) \mapsto\left(p_{1}, p_{2}^{\prime}, \sigma_{1}, \sigma_{2}^{\prime}\right)$, such that $\left(p_{2}, \sigma_{2}\right) \mapsto\left(p_{2}^{\prime}, \sigma_{2}^{\prime}\right)$ is in $P_{2}$.

In the description of the theory in this section, we omit the transition labels for simplicity. Thus, a computation sequence is simply a sequence of configurations.

The theory assumes an assertion language and uses relation $\sigma=\alpha$ to mean that the state $\sigma$ satisfies the assertion $\alpha$. For a configuration $\gamma=(p, \sigma)$, we write $\gamma \models \alpha$ for $\sigma \models \alpha$. An assertion is valid if it is satisfied by any state.

The formalization of the theory is based on the notion of assertion function. An assertion function of $\left(P_{1}, P_{2}\right)$ is a partial function

$$
I: \text { Point }_{P_{1}} \times \text { Point }_{P_{2}} \rightarrow \text { Assertion }
$$

mapping pairs of program points of $\left(P_{1}, P_{2}\right)$ to assertions, such that $I$ is defined on $\left(\operatorname{entry}\left(P_{1}\right), \operatorname{entry}\left(P_{2}\right)\right)$ and $\left(\operatorname{exit}\left(P_{1}\right), \operatorname{exit}\left(P_{2}\right)\right)$. This requirement is technical as one can always define $I$ on these pairs as $\top$. Assertions defined on such an $I$ are called inter-program assertions. Given a pair of points $\hat{p}$ and a pair of states $\hat{\sigma}$ of $\left(P_{1}, P_{2}\right)$, we say that $\hat{p}$ is $I$-observable if $I(\hat{p})$ is defined. For a configuration $\gamma=(\hat{p}, \hat{\sigma})$, we write $\gamma \models I$ if $I(\hat{p})$ is defined and $\hat{\sigma} \models I(\hat{p})$.

The theory introduces the notion of weakly-extendible assertion function as a wellsuited notion for describing inter-program properties.

DEFINITION 3.1. Let $I$ be an assertion function of a pair $\left(P_{1}, P_{2}\right)$ of programs. The function $I$ is weakly extendible if every run

$$
\gamma_{0}, \ldots, \gamma_{i}
$$

of $\left(P_{1}, P_{2}\right)$, such that $i \geq 0, \gamma_{0} \models I, \gamma_{i} \models I$, and $\gamma_{i}$ is not an exit configuration, can be extended to a run

$$
\gamma_{0}, \ldots, \gamma_{i}, \ldots, \gamma_{i+n}
$$

such that (1) $n>0$, and (2) $\gamma_{i+n} \models I$.

In [16] we show that, without appealing to the standard proof technique that uses wellfounded set, and using only inter-program assertions and the notion of weak extendibility, we can prove program equivalence and mutual simulations of two programs where one program has a loop that does not correspond to any loop in the other program, or even the loop is eliminated in the other program. For proving RCRs, we often encounter 
such a situation. For example, PIN is a scalar variable in the SPM, but is an array variable in the FSP. So, for checking and updating the PIN, the FSP contains loops that do not exist in the SPM.

We now develop verification conditions that guarantee weak extendibility. To this end, we need a notion of path of pairs of programs. A path $\pi$ of $\left(P_{1}, P_{2}\right)$ can be viewed as a trajectory in a two dimensional space: $\pi=\left(\pi_{1}, \pi_{2}\right)$, where $\pi_{1}$ is a path in the flow graph of $P_{1}$ and $\pi_{2}$ is a path in the flow graph of $P_{2}$. A path is trivial if it consists of a single pair of points. Given a path $\pi$ and an assertion $\psi$, we denote by $w p_{\pi}(\psi)$ and $w l p_{\pi}(\psi)$, respectively, the weakest and the weakest liberal preconditions of $\pi$ and $\psi$. Since we have to compute these preconditions, we assume that the programming language that we consider has the weak precondition property: for every path $\pi$ and every assertion $\psi, w p_{\pi}(\psi)$ exists and can effectively be computed. One can also compute $w l p_{\pi}(\psi)$ since it is equivalent to $w p_{\pi}(\psi) \vee \neg w p_{\pi}(\top)$. The precondition for paths of pairs of programs can also be derived from the precondition of paths of single programs.

DEFINITION 3.2. Let $I$ be an assertion function and $\Pi$ be a set of nontrivial paths such that, for every $\pi \in \Pi$, we have $\operatorname{start}(\pi)$ and $\operatorname{end}(\pi)$ to be $I$-observable. Denote by $\left.\left.\Pi\right|_{(} p, p^{\prime}\right)$ the set of paths in $\Pi$ whose first pair of points is $\left(p, p^{\prime}\right)$.

The weak verification condition $\mathbb{W}$ associated with $I$ and $\Pi$ consists of assertions of the form

$$
I(\operatorname{start}(\pi)) \Rightarrow w \operatorname{lp}_{\pi}(I(\operatorname{end}(\pi))),
$$

where $\pi \in \Pi$ and assertions of the form

$$
I(p) \Rightarrow \bigvee_{\left.\pi \in \Pi\right|_{\left(p, p^{\prime}\right)}} w p_{\pi}(\top)
$$

where $\left(p, p^{\prime}\right)$ is $I$-observable.

The first kind of assertion is a standard assertion for proving partial correctness of path. The second kind of assertion expresses that, whenever a configuration at $p$ satisfies $I(p)$, the computation from this configuration will inevitably follows at least one path in $\Pi$.

THEOREM 3.3. Let $\mathbb{W}, I$ and $\Pi$ be as in Definition 3.2 If every assertion in $\mathbb{W}$ is valid, then $I$ is weakly extendible.

The notion of weak verification condition is our notion for certificates that certify interprogram properties. In the next section we will use inter-program assertions to describe correspondences between observable variables. Later, to prove an RCR between two commands, one has to prove other inter-program properties between transition graphs of the commands. These program properties altogether describe the RCR. To prove such properties, we define an assertion function and prove that the function is weakly extendible. The certificates certifying these properties form a certificate for the RCR.

\section{Proving Representation Correspondences}

For our discussion on proving RCRs, we consider the application models $S$ and $T$ described in Section 2 To prove an RCR between $S$ and $T$, we are only concerned with 
command procedures, that is, for each corresponding command procedures, we prove an RCR between the procedures.

For two models $S$ and $T$, there is usually a one-to-one correspondence $O b s$ between global observable variables of $S$ and $T$ such that the values of each corresponding variables coincide at the entry and normal exit configurations of every command run. To this end, let us consider some command procedure $\mathrm{P}$. Let $O b_{p}, \mathrm{Obs}_{f}, \mathrm{Obs}_{a}$ be one-to-one correspondences specified for the end configurations of transitions emitting, respectively, a $\operatorname{Pass}_{P}$, a Fail $P$, an Abrupt $_{P}$ event. For simplicity of presentation, in the sequel let $O b s_{p}=O b s_{f}$. Let $O b s_{c}$ be a one-to-one correspondence specified for the start configurations of transitions emitting Call $_{P}$. We require that $O b s$ is included in $O b s_{p}$ and $O b s_{c}$. We say that a correspondence $f$ is included in a correspondence $g$ if for every mapping $x \mapsto y$ in $f$ is a mapping in $g$.

Denote by $P^{S}$ and $P^{T}$, respectively, the command $P$ in $S$ and in $T$. Given a function $f$, we denote by $\operatorname{dom}(f)$ the domain of $f$. For simplicity of notation, given a one-toone correspondence $g$, we abbreviate the assertion $\bigwedge_{x \in \operatorname{dom}(g)} x=g(x)$ to simply $g$. To prove an RCR between $P^{S}$ and $P^{T}$, we do the following steps:

1. Let $\alpha$ be an assertion, such that the assertion $\alpha \Rightarrow O b s_{c}$ is valid. That is, $\alpha$ describes the correspondence $O b s_{c}$. The assertion $\alpha$ can also describe invariants specific to $S$ or $T$. We prove that $\alpha$ is satisfied by the initializations of global variables.

2. We assert $\alpha$ at $\left(\operatorname{entry}\left(\mathbf{G}_{P^{S}}^{n}\right), \operatorname{entry}\left(\mathbf{G}_{P^{T}}^{n}\right)\right)$ and $\alpha^{\prime}$ at $\left(\operatorname{exit}\left(\mathbf{G}_{P^{S}}^{n}\right), \operatorname{exit}\left(\mathbf{G}_{P^{T}}^{n}\right)\right)$ such that the assertions $\alpha^{\prime} \Rightarrow O b s_{p}$ and $\alpha^{\prime} \Rightarrow \alpha$ are valid. That is, we assume that the correspondence expressed by $\alpha$ holds in the entry configurations of the procedures, and is preserved in the exit configurations.

3. Let $\psi, \psi^{\prime}$ be assertions asserted at, respectively, $\left(\operatorname{entry}\left(\mathbf{G}_{P^{S}}^{a}\right), \operatorname{entry}\left(\mathbf{G}_{P^{T}}^{a}\right)\right)$ and $\left(\operatorname{exit}\left(\mathbf{G}_{P^{S}}^{a}\right), \operatorname{exit}\left(\mathbf{G}_{P^{T}}^{a}\right)\right)$ such that the assertion $\psi^{\prime} \Rightarrow O b s_{a}$ is valid. That is, the correspondence $O b s_{a}$ holds when procedure runs terminate abruptly.

4. We prove that for every finite run of $\mathbf{G}_{P^{T}}^{n}$, there is a finite run of $\mathbf{G}_{P^{S}}^{n}$ from configurations satisfying $\alpha$, and vice versa, such that the final configurations of the runs satisfy the assertion $\psi$.

One can demonstrate (1) easily since it amounts to proving that the initializations of global variables satisfy $\alpha$. In the sequel we focus on the steps (2), (3), and (4).

We present our proof technique for proving RCRs of commands by means of a real example of a command called checkPIN that is used for authenticating users. In this paper we only consider proving RCRs between the SPM and the FSP of the command. Proving RCRs between the FSP and the TDS follows the same steps above. The SPM is written in a domain-specific language, called command description language, that resembles a subset of Java. Each command can be thought of as a method that has clauses: one pass clause describing conditions and state updates of successful completion of a run of the command; one or more fail clauses describing logic failures and the corresponding state updates; and one abrupt clause describing abrupt behavior of the command. For each command procedure $P$, the pass and fail clauses of the command constitute the transition graph $\mathbf{G}_{P}^{n}$, while the abrupt clause constitutes the transition graph $\mathbf{G}_{P}^{a}$. 


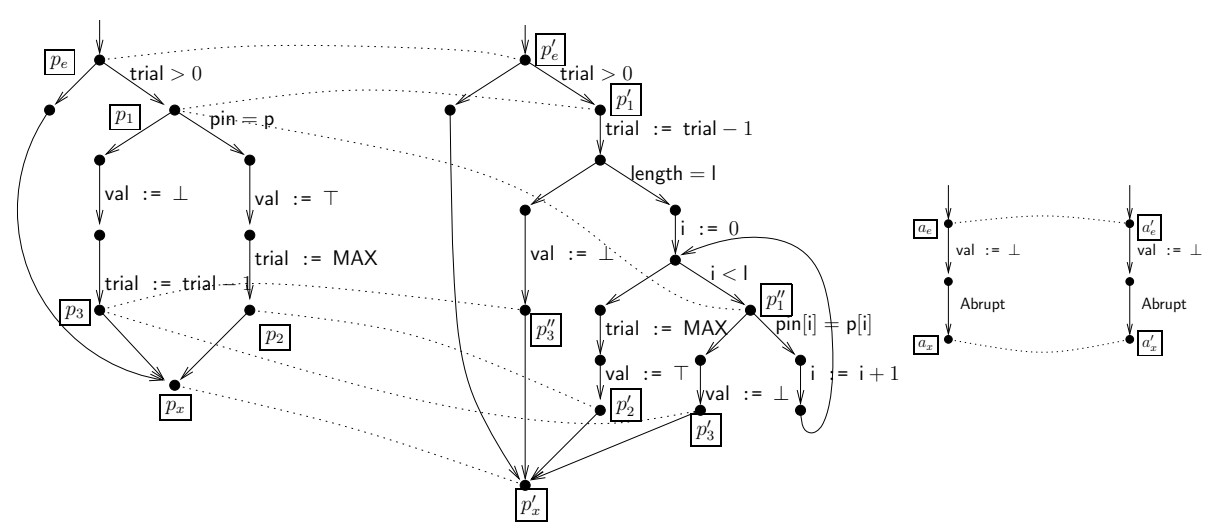

Fig. 1. SPM and FSP of checkPIN

The FSP is written in a subset of Java. Each command procedure $P$ is a method of the form:

\section{$P(\ldots)\{\operatorname{try}\{\ldots\} \operatorname{catch}($ CardTearException) $\{\ldots\}\}$}

The try part constitutes $\mathbf{G}_{P}^{n}$, while the catch part constitutes $\mathbf{G}_{P}^{a}$. Details of SPM and FSP can be found in our technical report [12].

EXAMPLE 4.1. We prove that there is an RCR between two corresponding commands procedure $P_{c}$ by considering their transition graphs $\mathbf{G}_{P_{c}}^{n}$ and $\mathbf{G}_{P_{c}}^{a}$ separately. The lefthand pair of transition graphs in Figure 1 is $\mathbf{G}_{\text {checkPIN }}^{n}$ of the SPM, on the left of the pair, and $\mathbf{G}_{\text {checkPIN }}^{n}$ of the FSP, on the right of the pair. As a shorthand, we call the former $P_{1}$ and the latter $P_{1}^{\prime}$. For disjointness, we assume that all variables in $P_{1}^{\prime}$ are primed.

First the global variables of the SPM that we want to observe are trial, pin, val, and MAX. They correspond to their primed counterparts in the FSP. Additionally, at the entries of $P_{1}$ and $P_{1}^{\prime}$, the input pin $\mathrm{p}$ corresponds to $\mathrm{p}^{\prime}$, and at the exits of $P_{1}$ and $P_{1}^{\prime}$, the event variable $\varepsilon$ corresponds to $\varepsilon^{\prime}$. Next, we have to define the equality between scalar PIN and array PIN. Every array PIN $p$ is associated with a length $l$; we write this association as $(p, l)$. We introduce predicate $\equiv$ between such pairs such that, given array PINs $(p, l)$ and $\left(p^{\prime}, l^{\prime}\right)$, we say that $(p, l) \equiv\left(p^{\prime}, l^{\prime}\right)$ if $l=l^{\prime}, l \geq 0$, and for all $i=0, \ldots, l-1$, we have $p[i]=p^{\prime}[i]$. We introduce a predicate $\sim$ which is axiomatized as follows: for every scalar PINs $w, x$ and for every array PINs $y, z$,

$$
x \sim y \Rightarrow(y \equiv z \Leftrightarrow x \sim z) \quad x \sim y \Rightarrow(w=x \Leftrightarrow w \sim y) .
$$

The predicate $\sim$ defines the equality between a scalar PIN and an array PIN.

The following assertions express the correspondence between observable variables:

$$
\begin{aligned}
& \phi_{1} \Leftrightarrow \text { trial }=\text { trial }^{\prime} \quad \phi_{3} \Leftrightarrow \text { pin } \sim\left(\text { pin' }^{\prime} \text {, length' }\right) \quad \phi_{5} \Leftrightarrow \mathrm{p} \sim\left(\mathrm{p}^{\prime}, \mathrm{I}^{\prime}\right) \\
& \phi_{2} \Leftrightarrow \mathrm{val}=\mathrm{val}^{\prime} \quad \phi_{4} \Leftrightarrow \mathrm{MAX}=\mathrm{MAX}^{\prime} \quad \phi_{6} \Leftrightarrow \varepsilon=\varepsilon^{\prime}
\end{aligned}
$$


Next, we define an assertion function $I_{1}$ of $\left(P_{1}, P_{1}^{\prime}\right)$ as follows:

$$
\begin{aligned}
I_{1}\left(p_{e}, p_{e}^{\prime}\right)= & \bigwedge_{i=1}^{5} \phi_{i} \quad I_{1}\left(p_{x}, p_{x}^{\prime}\right)=\bigwedge_{i=1}^{6} \phi_{i} \\
I_{1}\left(p_{1}, p_{1}^{\prime}\right)= & \bigwedge_{i=1}^{5} \phi_{i} \wedge \text { trial }>0 \\
I_{1}\left(p_{1}, p_{1}^{\prime \prime}\right)= & \bigwedge_{i=2}^{5} \phi_{i} \wedge \text { trial }>0 \wedge \text { trial }=\text { trial }^{\prime}+1 \\
& \wedge_{\text {length }}^{\prime}=\mathrm{I}^{\prime} \wedge \mathrm{i}^{\prime}<\mathrm{I}^{\prime} \wedge\left(\forall j .0 \leq j<\mathrm{i}^{\prime} \Rightarrow \operatorname{pin}^{\prime}[j]=\mathrm{p}^{\prime}[j]\right) \\
I_{1}\left(p_{2}, p_{2}^{\prime}\right)= & \bigwedge_{i=1}^{5} \phi_{i} \wedge \mathrm{pin}=\mathrm{p} \wedge(\text { pin, length }) \equiv(\mathrm{p}, \mathrm{I}) \\
I_{1}\left(p_{3}, p_{3}^{\prime}\right)= & I_{1}\left(p_{3}, p_{3}^{\prime \prime}\right)=\bigwedge_{i=1}^{5} \phi_{i} \wedge \text { pin } \neq \mathrm{p} \wedge(\text { pin, length }) \not \equiv(\mathrm{p}, \mathrm{I})
\end{aligned}
$$

In this example, we prove an interesting part of RCR, that is, without any presence of card tears, for every run $R$ of $P_{1}$, there is a run $R^{\prime}$ of $P_{1}^{\prime}$ from compatible states, such that $R$ and $R^{\prime}$ are observationally equivalent. We denote by $\pi_{p, p^{\prime}}$ a path from $p$ to $p^{\prime}$, and by $\pi_{p}$ a trivial path consisting only of point $p$. We prove that $I_{1}$ is weakly extendible by the following reasoning. First, for every run of $\left(P_{1}, P_{1}^{\prime}\right)$ from an entry configuration that satisfies $I_{1}\left(p_{e}, p_{e}^{\prime}\right)$, the run can reach $\left(p_{1}, p_{1}^{\prime}\right)$ by following the path $\left(\pi_{p_{e}, p_{1}}, \pi_{p_{e}^{\prime}, p_{1}^{\prime}}\right)$ such that the end configuration satisfies $I_{1}\left(p_{1}, p_{1}^{\prime}\right)$. From this configuration, the run can be extended either by following the path $\left(\pi_{p_{1}, p_{3}}, \pi_{p_{1}^{\prime}, p_{3}^{\prime \prime}}\right)$ or by following the path $\left(\pi_{p_{1}}, \pi_{p_{1}^{\prime}, p_{1}^{\prime \prime}}\right)$ such that the end configuration satisfies $I_{1}$. From the configuration that satisfies $I\left(p_{1}, p_{1}^{\prime \prime}\right)$, the run can be extended either by following $\left(\pi_{p_{1}}, \pi_{p_{1}^{\prime \prime}, p_{1}^{\prime \prime}}\right)$, or by following $\left(\pi_{p_{1}, p_{2}}, \pi_{p_{1}^{\prime \prime}, p_{2}^{\prime}}\right)$, or by following $\left(\pi_{p_{1}, p_{3}}, \pi_{p_{1}^{\prime \prime}, p_{3}^{\prime}}\right)$. Without any of these paths, $I_{1}$ would not be weakly extendible. Thus, we have shown that, using the notion of weak extendibility, these paths show that the loop in $P_{1}^{\prime}$ terminates.

Note that every possible transition of $P_{1}$ is described by the nontrivial paths that constitute the first elements of all pairs of paths above. Therefore, we have proved that for every run $R$ of $P_{1}$, there is a run $R^{\prime}$ of $P_{1}^{\prime}$ from compatible states, such that $R$ and $R^{\prime}$ are observationally equivalent. We can use the same reasoning for proving the other direction. Indeed, by taking the set of all the above pairs of paths, one can prove that all assertions of the weak verification condition associated with $I_{1}$ and the set are valid.

Consider now the righthand pair of transition graphs in Figure 1 is $\mathbf{G}_{\text {checkPIN }}^{a}$ of the SPM, on the left of the pair, and $\mathbf{G}_{\text {checkPIN }}^{a}$ of the FSP on the right of the pair. As a shorthand, we call the former $P_{2}$ and the latter $P_{2}^{\prime}$. The SPM and FSP only have to guarantee that the validation status is set to false in case of power loss. That is, the only observable variables are val and its primed counterpart.

We define an assertion function $I_{2}$ of $\left(P_{2}, P_{2}^{\prime}\right)$ such that we have $I_{2}\left(a_{e}, a_{e}^{\prime}\right)=\top$ and $I_{2}\left(a_{x}, a_{x}^{\prime}\right)=(\mathrm{val}=\mathrm{val})$. It is easy to that $I_{2}$ is weakly extendible, which means that if a card tear occurs and the configurations of the runs at $\left(a_{e}, a_{e}^{\prime}\right)$ satisfies $I_{2}$, then both runs will emit the same event, which is Abrupt $_{\text {checkPIN }}$ and they both terminate in compatible states.

Finally we have to prove that for every finite run of $P_{1}$ with end state $\sigma$, there is a finite run of $P_{1}^{\prime}$ with end state $\sigma^{\prime}$, and vice versa, such that $\left(\sigma, \sigma^{\prime}\right)$ satisfies $I_{2}\left(a_{e}, a_{e}^{\prime}\right)$. Since $I_{2}\left(a_{e}, a_{e}^{\prime}\right)$ is satisfied by every state, then we have finished our proof.

Proving RCRs between an FSP and a TDS is challenging due to the features of the language of the TDS. A TDS is written in a subset of Java Card [15], which includes transient and persistent memory as well as transaction mechanism. When a card tear occurs, data stored in persistent memory will be kept in the memory, while those stored 
in transient memory will be lost. Variables whose values are stored in persistent memory are called persistent variables, while those whose values are stored in transient memory are called transient variables.

Transactions are managed by methods beginTransaction, commitTransaction, and abortTransaction with standard functionalities. The depth of a transaction is at most 1 . When a transaction is in progress, the updates of persistent variables are conditional, in the sense that the updates will be materialized if commitTransaction is called. Regardless a transaction is in progress or not, the updates of transient variables are unconditional. To model card tears and transactions, we use the desugaring method in [9]. Each command in the TDS is a Java method, and desugaring the command means translating the method into the same form as that of the FSP, that is, the method has a big try-catch construct. The catch construct sets all transient variables to their default values, and cancel the updates of persistent variables if the card tear occurs when a transaction is in progress.

One might have to relax Definition 2.2 of RCRs to prove RCRs between an FSP and a TDS. Let us consider the following toy commands:

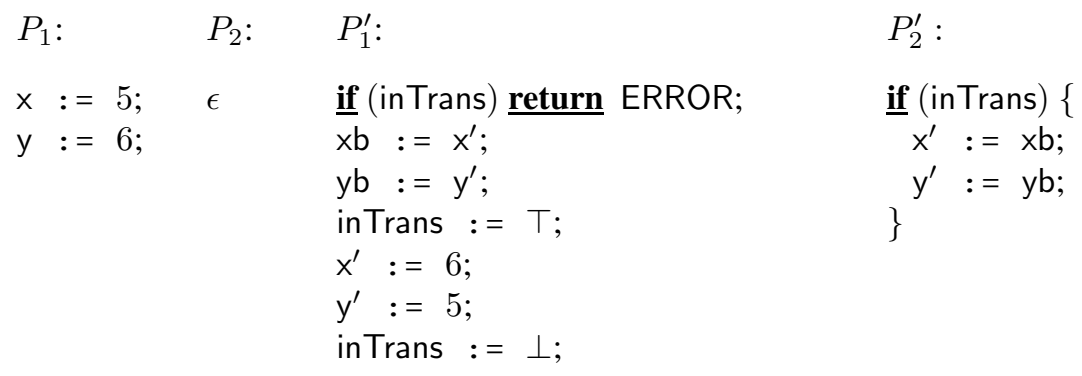

The programs (or transition graphs) $P_{1}$ and $P_{2}$ constitute the try and catch parts of the FSP, respectively. ( $P_{2}$ has no instruction.) Similarly for $P_{1}^{\prime}$ and $P_{2}^{\prime}$ of the desugared form of the TDS. Suppose that $x^{\prime}$ and $y^{\prime}$ are observable persistent variables that correspond to $x$ and $y$, respectively. The variables $x b$ and $y b$ are back-up variables for $x^{\prime}$ and $\mathrm{y}^{\prime}$. The boolean variable inTrans indicates whether a transaction is in progress or not; assume that it is false at the entry of $P_{1}^{\prime}$. In case of abrupt terminations, we want to ensure that the above correspondence holds. To this end, we have to assert at the entries of $P_{2}$ and $P_{2}^{\prime}$ the assertion $\phi$ below:

$$
\left(\neg \text { inTrans } \Rightarrow x=x^{\prime} \wedge y=y^{\prime}\right) \wedge(\text { inTrans } \Rightarrow x=x b \wedge y=y b)
$$

For every finite run $R^{\prime}$ of $P_{1}^{\prime}$ from a state satisfying inTrans $=\perp$, there is a finite run $R$ of $P_{1}$ such that the final configurations of the runs satisfy $\phi$. For example, if $R^{\prime}$ reaches the middle of transaction, e.g., the entry of $\mathrm{y}^{\prime}:=6$, then $R$ simply stays at the entry of $P_{1}$. However, showing the other way around is not possible. When a run $R$ reaches the entry of y $:=6$, then there is no finite run $R^{\prime}$ of $P_{1}^{\prime}$ such that the final configurations satisfy $\phi$. Thus, according to Definition 2.2 there is no RCR between the commands.

To handle such an above case, one can relax Definition 2.2. That is, we only require that for every run $R$ of $P^{T}$ from a configuration $\gamma$, there is a run $R^{\prime}$ of $P^{S}$ from a configuration $\gamma^{\prime}$, where $\gamma$ and $\gamma^{\prime}$ are compatible, such that $R$ and $R^{\prime}$ are observationally equivalent. The drawback of this relaxed definition is that if $P^{T}$ does not terminate 
and the assertion at the entries of abrupt graphs is valid, then there is always an RCR between $P^{T}$ and $P^{S}$. Nevertheless, with this relaxed definition, we can still preserve security properties for $S$ in $T$, as shown in the following section.

\section{Property Preservation}

In this section we show how security properties of the SPM can be preserved in the FSP using RCRs. Property preservation between the FSP and the TDS can be explained in the same way. We are only concerned with security properties that can be characterized as partial correctness properties: a procedure $P$ is partially correct with respect to a precondition $\alpha$ and a postcondition $\beta$, denoted by $\{\alpha\} P\{\beta\}$, if for every run of $P$ from a state satisfying $\alpha$ and reaching an exit configuration, this configuration satisfies $\beta$.

Consider again the application models $S$ and $T$ and the one-to-one correspondences $O b s_{p}, O b s_{f}, O b s_{a}, O b s_{c}$ described at the beginning of Section 4 . We show property preservation by the following theorem:

THEOREM 5.1. Let $\alpha$ and $\beta$ be, respectively, a precondition and a postcondition for a procedure $P^{S}$ such that $\{\alpha\} P^{S}\{\beta\}$. Let $\alpha^{\prime}$ and $\beta^{\prime}$ be, respectively, a precondition and a postcondition for a procedure $P^{T}$ such that the assertions

$$
\begin{aligned}
& O \operatorname{Obs}_{c} \Rightarrow\left(\alpha \Leftrightarrow \alpha^{\prime}\right) \\
& \left(O b s_{p} \wedge \varepsilon=\operatorname{Pass}_{P}\right) \vee\left(O b s_{f} \wedge \varepsilon=\text { Fail }_{P}\right) \vee\left(O b s_{a} \wedge \varepsilon=\operatorname{Abrupt}_{P}\right) \Rightarrow\left(\beta \Leftrightarrow \beta^{\prime}\right)
\end{aligned}
$$

are valid. If there is an RCR between $P^{S}$ and $P^{T}$, then $\left\{\alpha^{\prime}\right\} P^{T}\left\{\beta^{\prime}\right\}$.

As an example, consider again the command and assertions in Example 4.1 Suppose that the property that we want to preserve is as follows: for any run of checkPIN, the value of variable val at the exit configuration of the run is true if and only if the run

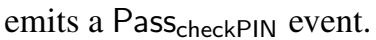

Let $\psi$ be the assertion (val $=\top \Leftrightarrow \varepsilon=$ Pass $_{\text {checkPIN }}$ ) and $\varphi$ be the conjunction of the following assertions: (1) $\mathrm{MAX}>0$, (2) $0 \leq$ trial $\leq \mathrm{MAX}$, and (3) trial $<$ $\mathrm{MAX} \Rightarrow \mathrm{val}=\perp$. The above property can be expressed as a partial correctness property $\{\varphi\}$ checkPIN $\{\psi\}$. One can use standard Floyd-Hoard proof technique [68] to prove the property for both the SPM and the FSP.

Suppose that we have proved that the property holds for the SPM. We have shown in Example 4.1 that there is an RCR between the command checkPIN of the SPM and of the FSP. Let $P$ be the command checkPIN in the FSP. Recall again the assertions $\phi_{1}, \ldots, \phi_{6}$ in the example. Given an assertion $\alpha$, let us denote by $p(\alpha)$ the assertion obtained from $\alpha$ by replacing each variable in $\alpha$ with its primed notation. Now, since the following assertions

$$
\begin{aligned}
& \bigwedge_{i=1}^{5} \phi_{i} \Rightarrow(\varphi \Leftrightarrow p(\varphi)) \\
& \left(\bigwedge_{i=1}^{6} \phi_{i} \wedge\left(\varepsilon=\operatorname{Pass}_{P} \vee \varepsilon=\text { Fail }_{P}\right)\right) \vee\left(\phi_{2} \wedge \varepsilon=\text { Abrupt }_{P}\right) \Rightarrow(\psi \Leftrightarrow p(\psi))
\end{aligned}
$$

are valid, then by Theorem5.1 we have $\{p(\varphi)\} P\{p(\psi)\}$. 


\section{Related Work and Conclusion}

We developed a method for proving RCRs in the CC EAL7 certification of smart-card applications. We presented a modelling framework by which the representations of the SPM, the FSP, and the TDS can be modelled uniformly. Our framework is an extension of the modelling framework of procedural programs in [18], in the sense that we model abrupt behavior of procedures. Our definition of RCRs is mutual simulations between two application models. We apply the theory of inter-program properties in [16] for proving RCRs and providing certificates about them. The theory has been used for proving properties in translation validation approach to compiler verification [13 19]16]. In this paper we have shown another venue for the application of the theory. The application is beneficial since the theory provides a notion of certificate, which is essential in the CC EAL7 certification.

There have been a few works on formal specification and verification in the CC framework; closely related to ours is [4]. Their work is based on B method. Their definition of RCRs is similar to ours, in the sense that, for each command, they have a mapping between input-output relationships of two application models. Their work does not address complex data abstractions like our PIN, and their commands do not contain loops. However, their work has gone beyond ours in the sense that they included a model of Java Card API for APDU commands [15].

Another related work is by Heitmeyer et. al. on verifying enforcement of data separation in the kernel of a software-based embedded device [7]. Similar to ours, their work uses a state machine model consisting of events as a specification. Concrete code is partition into event code, trusted code, and other code. Event code corresponds to an event in the state-machine specification and such code is annotated with preconditions and postconditions. Their work construct two mappings: one is between events of the state machine and of the code, and the other is between assertions describing preconditions and postconditions of corresponding events. RCRs are proved for each corresponding events, that is, the precondition and the postcondition of an event in the code imply, respectively, the precondition and postcondition of the corresponding event in the specification. In their work, event code contains no loops, and they do not prove the relation between the code and its precondition and postcondition. Moreover, the mapping between assertions is based only on syntactic matching. Unlike ours, their work deals with real C code.

Other works on the CC certification have not addressed RCRs, or have only given little efforts on RCRs [3|17]. One distinguish feature in our work that has not been addressed by others is proving property preservation using RCRs.

There has been some work related to the specification and verification of smartcard applications, but not in the CC certification. Paper [14] describes a verification of Mondex electronic purse based on abstract state machine (ASM). The work is not in the $\mathrm{CC}$, but it uses a notion of refinement simulation between ASMs to show correctness of a concrete implementation. The operations (similar to commands) in Mondex are simple and contains no loops and no complex data abstractions. The work in [2] describes a case study in the specification and verification of an electronic purse application. The work is concerned only with the specification and verification of commands in the implementation code. The work can complement our work in proving properties of the implementation code. 
In this paper we do not address RCRs between the TDS and the implementation code. We assume that existing work on certified and certifying compilers [11]13] can be used to provide RCRs between the TDS and the implementation code. We are currently developing certification tools based on the method described in this paper. We take JML approach [10] to specifying assertion function. That is, we use special comments to put labels denoting program points in the programs, and write the assertion function in a separate file. We use off-the-shelf data-flow analyses, such as global value numbering, to assist users in defining assertion functions, so that users only concentrate on one-toone correspondences between observable variables. We are developing heuristics based on observable events to alleviate the burdens of specifying paths in weak verification conditions; this is the topic of our future work. Assertions in the verification conditions can then be proved using SMT solvers, such as Yices [5].

\section{References}

1. Common Criteria for Information Technology Security Evaluation, Version 3.1, CCMB2007-09-003 (2007)

2. Breunesse, C.-B., Cataño, N., Huisman, M., Jacobs, B.: Formal methods for smart cards: an experience report. Sci. Comput. Program. 55(1-3), 53-80 (2005)

3. Chetali, B., Nguyen, Q.-H.: Industrial use of formal methods for a high-level security evaluation. In: Formal Methods, pp. 198-213 (2008)

4. Dadeau, F., Potet, M.-L., Tissot, R.: A B formal framework for security developments in the domain of smart card applications. In: Security Conference, pp. 141-155 (2008)

5. Dutertre, B., de Moura, L.M.: A fast linear-arithmetic solver for DPLL(T). In: Ball, T., Jones, R.B. (eds.) CAV 2006. LNCS, vol. 4144, pp. 81-94. Springer, Heidelberg (2006)

6. Floyd, R.W.: Assigning meaning to programs. In: Schwartz, J.T. (ed.) Proceedings of Symposium in Applied Mathematics, pp. 19-32 (1967)

7. Heitmeyer, C.L., Archer, M., Leonard, E.I., McLean, J.: Formal specification and verification of data separation in a separation kernel for an embedded system. In: CCS 2006: Proceedings of the 13th ACM conference on Computer and communications security, pp. 346-355. ACM, New York (2006)

8. Hoare, C.A.R.: An axiomatic basis for computer programming. CACM 12(10), 576-580 (1969)

9. Hubbers, E.-M.G.M., Poll, E.: Reasoning about card tears and transactions in Java Card. In: Wermelinger, M., Margaria-Steffen, T. (eds.) FASE 2004. LNCS, vol. 2984, pp. 114-128. Springer, Heidelberg (2004)

10. Leavens, G., Cheon, Y.: Design by contract with JML (2003)

11. Leroy, X.: Formal certification of a compiler back-end or: programming a compiler with a proof assistant. SIGPLAN Not. 41(1), 42-54 (2006)

12. Narasamdya, I., Périn, M.: Certification of smart-card applications in common criteria. Technical Report TR-2008-14, Verimag (September 2008)

13. Rinard, M., Marinov, D.: Credible compilation with pointers. In: Proceedings of the FLoC Workshop on Run-Time Result Verification, Trento, Italy (July 1999)

14. Schellhorn, G., Grandy, H., Haneberg, D., Reif, W.: The mondex challenge: Machine checked proofs for an electronic purse. In: Misra, J., Nipkow, T., Sekerinski, E. (eds.) FM 2006. LNCS, vol. 4085, pp. 16-31. Springer, Heidelberg (2006) 
15. Sun Micro systems, Inc, Palo Alto, California. Java Card 3.0 Platform Specification (2008), http: //java.sun.com/javacard/3.0/

16. Voronkov, A., Narasamdya, I.: Proving inter-program properties. Technical Report TR-200813, Verimag (2008)

17. Wilding, M., Greve, D.A., Hardin, D.: Efficient simulation of formal processor models. Formal Methods in System Design 18(3), 233-248 (2001)

18. Zaks, A., Pnueli, A.: CoVaC: Compiler validation by program analysis of the cross-product. In: Cuellar, J., Maibaum, T., Sere, K. (eds.) FM 2008. LNCS, vol. 5014, pp. 35-51. Springer, Heidelberg (2008)

19. Zuck, L.D., Pnueli, A., Goldberg, B.: VOC: A methodology for the translation validation of optimizing compilers. J. UCS 9(3), 223-247 (2003) 